\title{
Spectral editing based on selective excitation and Lee-Goldburg cross-polarization under magic angle spinning
}

\author{
Shing-Jong Huang ${ }^{\mathrm{a}}$, Yao-Hung Tseng ${ }^{\mathrm{b}}$, Yun Mou ${ }^{\mathrm{b}}$, Shang-Bin Liu ${ }^{\mathrm{a}}$, Shih-Hao Huang ${ }^{\mathrm{c}}$, \\ Chun-Pin Lin ${ }^{c}$, Jerry C.C. Chan ${ }^{b, *}$ \\ ${ }^{a}$ Institute of Atomic and Molecular Sciences, Academia Sinica, Taipei, Taiwan \\ ${ }^{\mathrm{b}}$ Department of Chemistry, National Taiwan University, Taipei, Taiwan \\ ${ }^{\mathrm{c}}$ Graduate Institute of Clinical Dentistry, National Taiwan University, Taipei, Taiwan
}

Received 19 July 2005; received in revised form 1 October 2005

Available online 7 November 2005

\begin{abstract}
We show that a Gaussian-shaped pulse can be used to excite selected ${ }^{1} \mathrm{H}$ signals in hydroxyapatite, monetite and $\mathrm{H}-\mathrm{Y}$ zeolite loaded with trimethylphosphine oxide (TMPO). This selective excitation method can be incorporated into Lee-Goldburg (LG) crosspolarization to obtain useful spectral editing opportunity. This new strategy has been applied to identify the Brønsted and the Lewis acid sites in $\mathrm{H}-\mathrm{Y}$ zeolite using TMPO as the probe molecule.
\end{abstract}

(C) 2005 Elsevier Inc. All rights reserved.

Keywords: MAS; Solid-state NMR; Lee-Goldburg; Zeolite; HY; TMPO; LG-CP

\section{Introduction}

Cross-polarization (CP) under magic-angle spinning (MAS) is a ubiquitous technique in solid-state NMR [1]. While CP is a valuable method to enhance signal intensity [2], its application in probing heteronuclear spin-pair interaction is not satisfying because of the spin diffusion during the contact time. Although the ${ }^{1} \mathrm{H}$ homonuclear decoupled CP technique has been demonstrated for a long time [3], this sophisticated multiple-pulse based approach, which can suppress spin diffusion during the contact time period, has not been widely applied because of the rather involved set-up procedure. Recently, it has been shown that the Lee-Goldburg (LG) homonuclear decoupling technique can be combined with $\mathrm{CP}$ to achieve polarization transfer with efficient suppression of ${ }^{1} \mathrm{H}-{ }^{1} \mathrm{H}$ spin diffusion $[4,5]$. This novel LG-CP technique, which was originally developed for ${ }^{1} \mathrm{H}-{ }^{13} \mathrm{C}$ multiple-spin systems, is easy to

\footnotetext{
*Corresponding author. Chemistry Department, National Taiwan University, No. 1, Section 4, Roosevelt Road, Taipei, Taiwan. Fax: $+886223636359$

E-mail address: chanjcc@ntu.edu.tw (J.C.C. Chan).
}

implement and has been applied to obtain the ${ }^{1} \mathrm{H}-{ }^{31} \mathrm{P}$ HETCOR spectra for silica alumina loaded with trimethylphosphine oxide (TMPO) molecules [6]. We have also demonstrated that the LG-CP HETCOR method can be applied for the study of apatite minerals formed on the surface of bioactive glasses [7].

In view of the applicability of LG-CP in ${ }^{1} \mathrm{H}_{-}{ }^{31} \mathrm{P}$ multiplespin system, it is interesting to further explore its potential in spectral editing. One interesting issue in the subject of heterogeneous catalysis is the identification of the Brønsted and the Lewis acid sites on silica-alumina surfaces. A useful tactics is to compare the relative intensities of the ${ }^{31} \mathrm{P}$ resonances when the sample loaded with TMPO is subjected to various degrees of hydration treatments $[8,9]$. In this work, we show that LG-CP combined with excitation or suppression of selected ${ }^{1} \mathrm{H}$ signal can provide a versatile spectral editing opportunity for the discernment of the Brønsted and the Lewis acid sites in TMPO-loaded silica-alumina. Provided that the ${ }^{1} \mathrm{H}$ signal loss during the selective excitation is not significant, this one-dimensional (1D) NMR technique is preferred over the LG-CP HETCOR approach for the studies of TMPO loaded systems with very limited amount of acid sites. 


\section{Experimental}

\subsection{Sample preparation}

The crystalline standards hydroxyapatite (Sigma-Aldrich), monetite (Acros Organics) and TMPO (Alfa Chemicals) were used as received. $\mathrm{H}-\mathrm{Y}$ zeolite was obtained by means of thrice ion exchange of a commercial $\mathrm{Na}-\mathrm{Y}$ zeolite (Strem Chemicals, $\mathrm{Si} / \mathrm{Al}=2.6$ ) with excess $1 \mathrm{M} \mathrm{NH} \mathrm{NHO}_{3}$ aqueous solution. The sample was then calcined in air at $813 \mathrm{~K}$ and dehydrated under vacuum at $673 \mathrm{~K}$ for $48 \mathrm{~h}$. The desired amount of TMPO was loaded into the dehydrated $\mathrm{H}-\mathrm{Y}$ sample using purified dichloromethane as solvent under dry $\mathrm{N}_{2}$ atmosphere. After solvent evaporation under vacuum, the TMPO-loaded sample was further subjected to baking treatment at $423 \mathrm{~K}$ for $1 \mathrm{~h}$ to ensure homogeneous adsorption of the TMPO molecules. The sample was packed under $\mathrm{N}_{2}$ atmosphere for NMR measurements.

\subsection{Solid-state NMR}

All NMR experiments were carried out at ${ }^{31} \mathrm{P}$ and ${ }^{1} \mathrm{H}$ frequencies of 121.5 and $300.1 \mathrm{MHz}$, respectively, on a Bruker DSX300 NMR spectrometer equipped with a commercial $4 \mathrm{~mm}$ probe. The spectra were measured at room temperature. The sample was confined to the middle $1 / 3$ of the rotor volume using Teflon spacers. MAS frequency was set at $10 \mathrm{kHz}$ with variation limited to $\pm 3 \mathrm{~Hz}$ using a commercial pneumatic control unit. Chemical shifts were externally referenced to $85 \%$ phosphoric acid and TMS for ${ }^{31} \mathrm{P}$ and ${ }^{1} \mathrm{H}$, respectively. Selective Gaussian pulses were 2 or $4 \mathrm{~ms}$ long. The LG-CP contact time was $4 \mathrm{~ms}$, during which the ${ }^{1} \mathrm{H}$ nutation frequency and the resonance offset were set equal to 50 and $35.35 \mathrm{kHz}$, respectively, in order to fulfill the LG irradiation condition. Proton decoupling during the acquisition time was set to $50 \mathrm{kHz}$. Quadrature detection in the F1 dimension was achieved by the hypercomplex approach. For each $t_{1}$ increment 32 transients were accumulated, and a total of 63 increments were done at steps of twice the rotor period. Spin-temperature inversion was incorporated with CYCLOPS [10], resulting in an eight-step phase cycling.

\subsection{Numerical simulations}

All numerical studies in this work were carried out using SIMPSON (version 1.1.1) [11]. For our simulations, the maximum time step over which the Hamiltonian is approximated to be time-independent was set to $3.0 \mu \mathrm{s}$. Typically, a powder averaging scheme containing 100 REPULSION angles $(\alpha$ and $\beta$ ) [12] and $18 \gamma$ angles was chosen. Relaxation effects were ignored. Simulations were done for a linear three spin system constructed based on the structural parameters of the three closest protons in monetite [13], where two of the them have the same isotropic chemical shift of $13.6 \mathrm{ppm}$ and the remaining one is of $16.2 \mathrm{ppm}$ [14]. The distance between adjacent protons is of $3.76 \AA$ long. All the dipolar interactions among the three protons were considered. The anisotropies and the asymmetry parameters (according to the convention adopted in SIMPSON) of the chemical shift tensors of the three protons were arbitrarily set to be $(10 \mathrm{ppm}, 0.4),(11 \mathrm{ppm}, 0.0)$ and (9 ppm, 0.8). The orientations of the chemical shift tensors with respect to the dipolar framework were set arbitrarily. A Gaussian line broadening of $50 \mathrm{~Hz}$ was applied prior to the Fourier transformation of each calculated FID.

\section{Results and discussion}

\section{1. ${ }^{1} H$ selective excitation}

Recently, it has been shown that a Gaussian-shaped pulse [10] can be used for selective excitation under the condition of magic-angle spinning [15]. While this strategy has been successfully applied to ${ }^{13} \mathrm{C}$ or ${ }^{15} \mathrm{~N}$ multiple-spin systems, at the first glance it may appear difficult to achieve selective excitation in a ${ }^{1} \mathrm{H}$ multiple-spin system because of the strong homonuclear dipole-dipole interaction. Nevertheless, experimentally we found that it is possible to realize ${ }^{1} \mathrm{H}$ selective excitation under fast magic-angle spinning. Fig. 1a shows the results obtained for hydroxyapatite and monetite at a spinrate of $10 \mathrm{kHz}$. Referring to the ${ }^{1} \mathrm{H}$ spectrum of hydroxyapatite, the two ${ }^{1} \mathrm{H}$ signals at 0.2 and $5 \mathrm{ppm}$ originate from $\mathrm{OH}^{-}$groups and water molecules, respectively [14]. Since the ${ }^{1} \mathrm{H}$ signal of the $\mathrm{OH}^{-}$group is well resolved and inhomogeneously broadened [16], it is easy to invert or nullify this signal selectively.

Monetite has four proton sites and two of them are crystallographically equivalent [13]. The effectiveness of ${ }^{1} \mathrm{H}$ selective excitation in a multiple-spin system depends on the extent of ${ }^{1} \mathrm{H}$ spin diffusion during the excitation period, which in turn depends on the spinning frequency, the chemical shift dispersion, the homonuclear dipolar interaction and the spatial arrangement of the spin cluster. While it is not straightforward to quantify the effect of spin diffusion during the selective excitation, one may qualitatively evaluate its effect by comparing the signal intensity of the selectively excited species with that observed in the Bloch decay spectrum. Referring to Fig. 1b, although the ${ }^{1} \mathrm{H}$ spectrum of monetite has limited resolution, it remains possible to selectively suppress one of the spectral components without losing too much the intensity of another one. The ratio of the two selectively excited proton signal intensities is found to be 1:3.06, which is very similar to that determined for the Bloch decay spectrum (1:2.92). In this case, although the excitation is not a perfectly selective one, the effect of spin diffusion does not wash out the selectivity to any significant extent.

\subsection{Selective LG-CP HETCOR}

Since the LG irradiation has a prominent feature that homonuclear dipole-dipole interactions are suppressed, a 

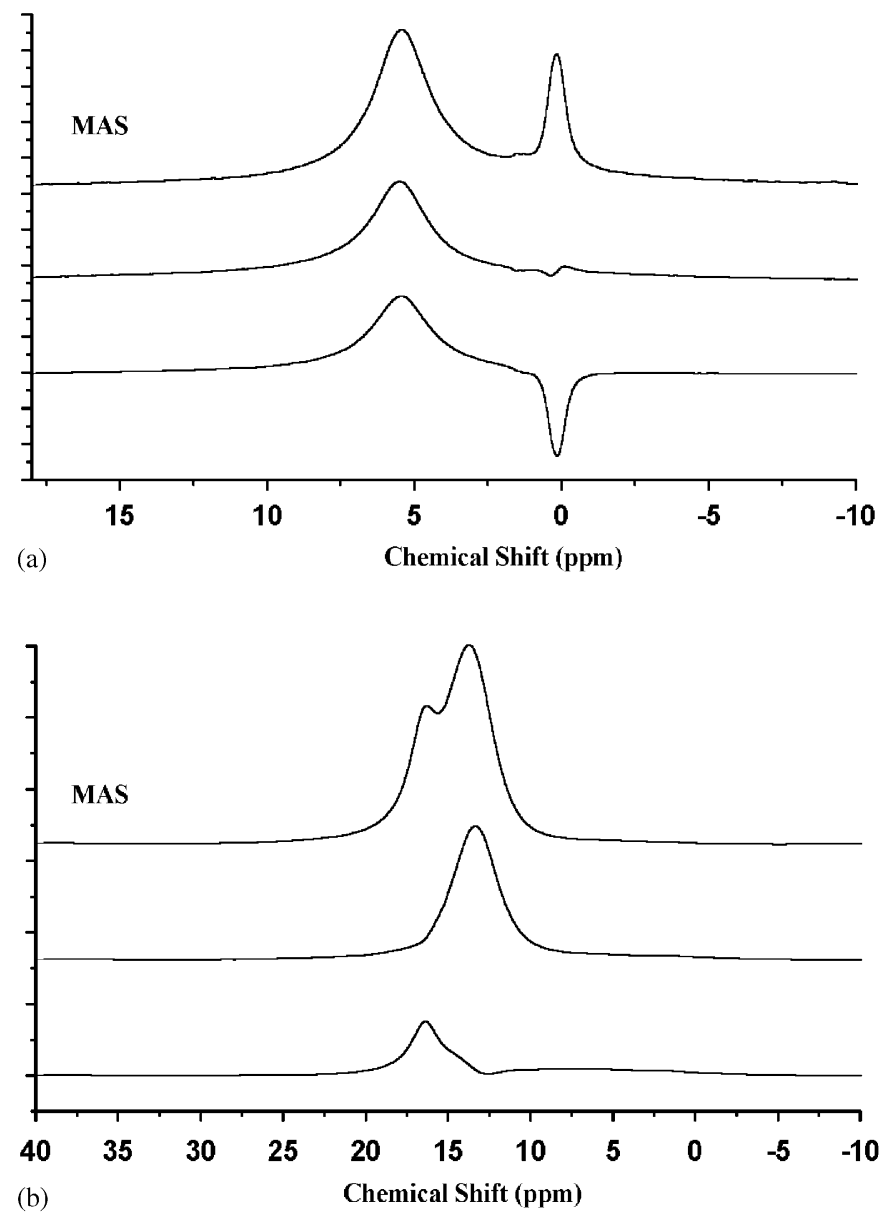

(b)

Chemical Shift (ppm)

Fig. 1. Selective ${ }^{1} \mathrm{H}$ excitation by Gaussian-shaped pulses under $10 \mathrm{kHz}$ MAS spinning: (a) the apatite signal of hydroxyapatite is nullified and inverted by selective $\pi / 2$ and $\pi$ pulses, respectively, and (b) the two spectral components of monetite are individually excited. The relative intensities of the selectively excited spectral components are very similar to those of the simple MAS spectrum (see the text).

very useful spectral editing opportunity is obtained when LG-CP is combined with the selective excitation of the source magnetization. Fig. 2 shows the pulse sequence of the resultant selective LG-CP HETCOR technique. The desired spectral component for polarization transfer is first selectively excited. The flip-angle of the pulse prior to the contact time is adjusted so that the excited magnetization is spin locked along the effective field of the LG irradiation. Consequently, the undesired spectral component of the source magnetization becomes perpendicular to the effective field without contributing to the $\mathrm{CP}$ signal. To demonstrate this idea experimentally, we attempt to suppress the apatite component of the ${ }^{31} \mathrm{P}\left\{{ }^{1} \mathrm{H}\right\}$ LG-CP HETCOR spectrum measured for a dentin sample obtained from mouse teeth, which has carbonated apatite as the major constituent. Fig. 3(a) shows the regular LGCP HETCOR spectrum, which is quite similar to the HETCOR spectrum measured for H5 animal bone [3]. To suppress the cross peak due to the apatite signal (the sharper cross peak), we selectively excite the apatite ${ }^{1} \mathrm{H}$

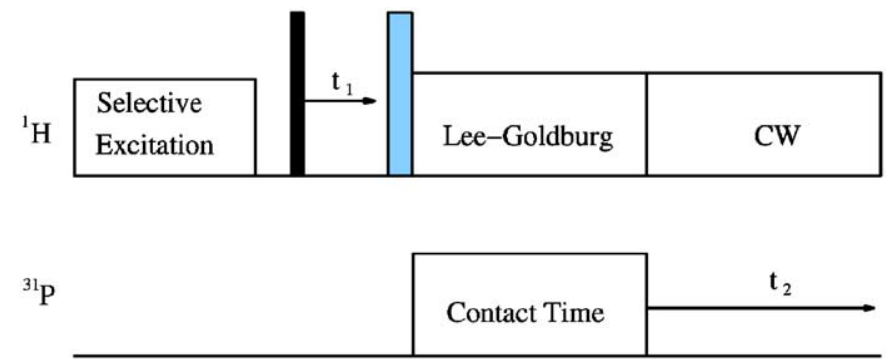

Fig. 2. Pulse sequence used for LG-CP HETCOR experiment. The block in black denotes a $\pi / 2$ pulse. The pulse denoted by the block in grey is used to place the excited magnetization along the effective field of the LG irradiation.
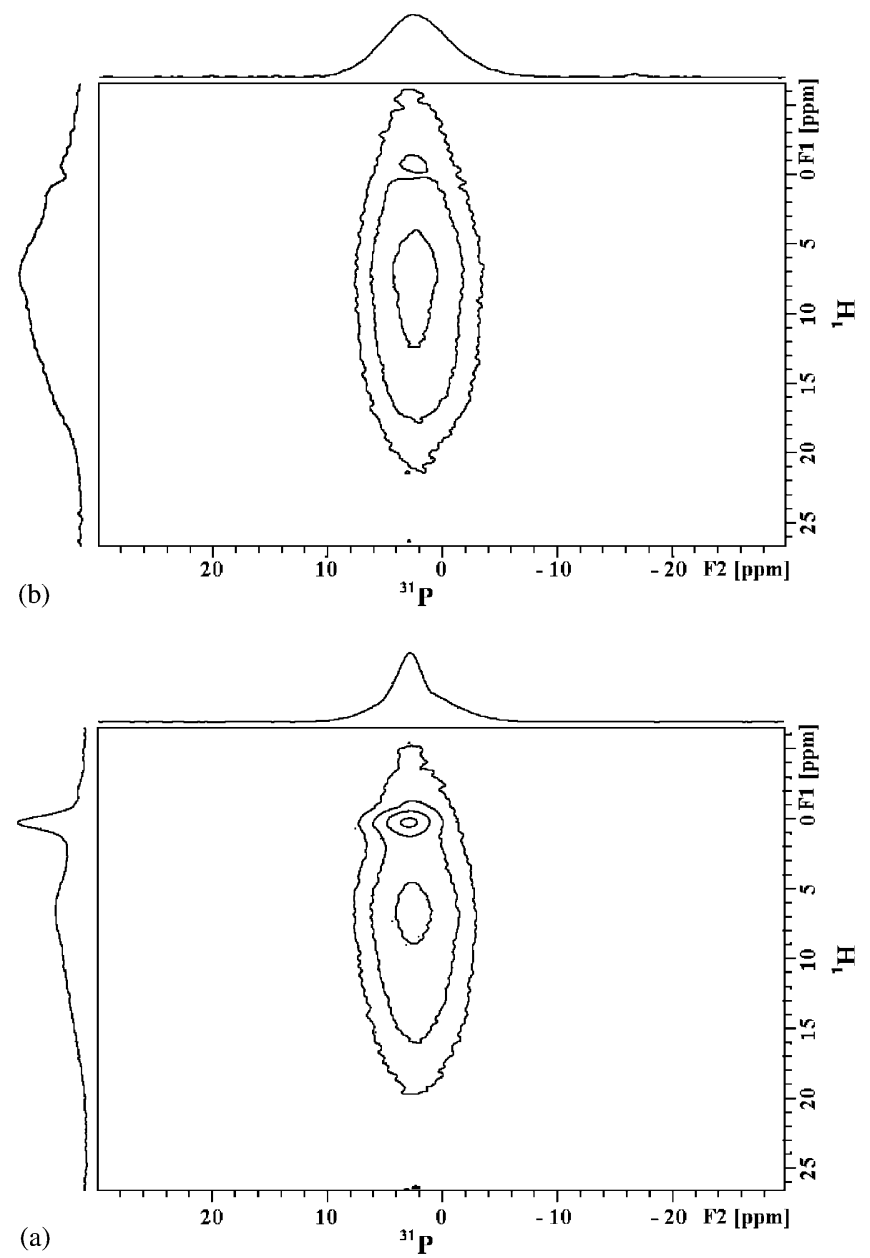

Fig. 3. ${ }^{31} \mathrm{P}\left\{{ }^{1} \mathrm{H}\right\}$ LG-CP HETCOR spectra measured for mouse dentin sample: (a) control and (b) the apatite signal is selectively suppressed before the cross-polarization.

signal by a soft Gaussian-shaped $\pi / 2$ pulse and then flip it back to the $z$-axis by a hard $\pi / 2$ pulse. The hence excited magnetization due to the water signal is spin locked for LG-CP polarization transfer. Fig. 3(b) shows that the suppression of the apatite component in the LG-CP HETCOR spectrum is successful. 
Although we have shown in the previous section that selective excitation of a ${ }^{1} \mathrm{H}$ multiple-spin system cannot be a perfect one, our LG-CP HETCOR spectrum in Fig. 3(b) proves that the selectivity given by a simple Gaussianshaped pulse is sufficient to provide a spectral editing opportunity for the subsequent LG-CP process. Consequently, one may derive a 1D NMR strategy by combining the idea of selective excitation with LG-CP to replace the LG-CP HETCOR approach (a 2D technique) for systems with very poor NMR sensitivity. Examples of such systems can be found in TMPO loaded silica system with low alumina content, where the ${ }^{31} \mathrm{P}$ signal due to the $\mathrm{CP}$ process between the bridging hydroxyl group and the attached TMPO molecule would be very weak.

\subsection{TMPO-loaded $H Y$}

The ${ }^{31} \mathrm{P}$ MAS spectrum of the TMPO-loaded HY sample is shown in Figs. 4. Based on the ${ }^{1} \mathrm{H} /{ }^{31} \mathrm{P} /{ }^{27} \mathrm{Al}$ TRAPDOR technique, it has been shown that the ${ }^{31} \mathrm{P}$ signals III $(55 \mathrm{ppm})$ and IV $(65 \mathrm{ppm})$ arise from TMPO interacting with Brønsted acid sites while the signal I (41 ppm) is due to the physisorbed TMPO [17]. While the signal V (75 ppm) is likely due to a new Brønsted acid site with stronger acidity, it is not trivial to assign the signal II ( $45 \mathrm{ppm})$. On the other hand, the two major spectral components of the ${ }^{1} \mathrm{H}$ spectrum are readily assigned as shown in Fig. 5. Given the excellent spectral resolution, a Gaussian-shaped pulse can be used to selectively suppress the ${ }^{1} \mathrm{H}$ signal at $2.1 \mathrm{ppm}$. Since the remaining ${ }^{1} \mathrm{H}$ signal at $7.2 \mathrm{ppm}$ is solely due to the hydroxyl group of the Brønsted acid site, the hence obtained ${ }^{31} \mathrm{P}\left\{{ }^{1} \mathrm{H}\right\}$ selective LG-CP spectrum can be compared with the regular LG-CP spectrum in order to help make the spectral assignment of the ${ }^{31} \mathrm{P}$ spectrum.

Fig. 6 shows the selective LG-CP, the regular LG-CP and their difference spectra measured for the TMPOloaded zeolite sample. As expected, the selective LG-CP spectrum has much lower intensity and therefore it is scaled up so that the intensity of signal $\mathrm{V}$ at $75 \mathrm{ppm}$ is nullified in

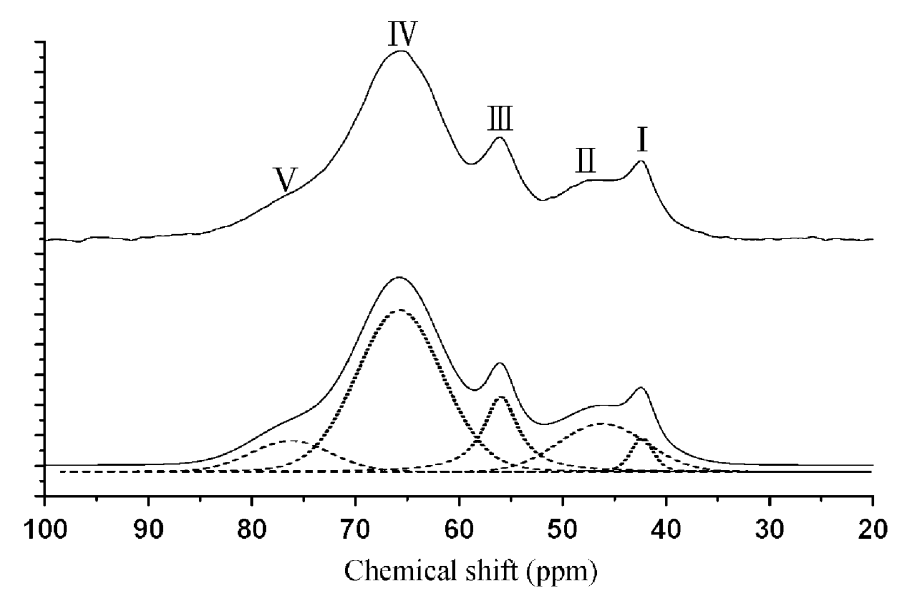

Fig. 4. ${ }^{31} \mathrm{P}$ MAS spectrum of the TMPO loaded $\mathrm{H}-\mathrm{Y}$ zeolite sample. The lower trace is the deconvolution with Gaussian functions.

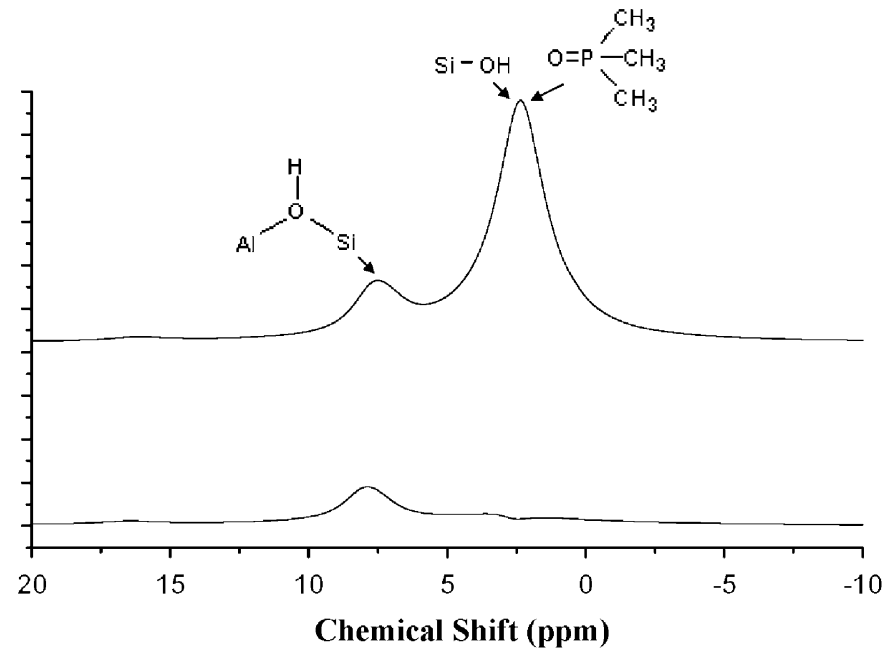

Fig. 5. ${ }^{1} \mathrm{H}$ spectrum of the TMPO loaded $\mathrm{H}-\mathrm{Y}$ zeolite sample. The upper trace is the regular MAS spectrum and the lower trace corresponds to the selective excitation of the bridging hydroxyl group (Brønsted acid site).

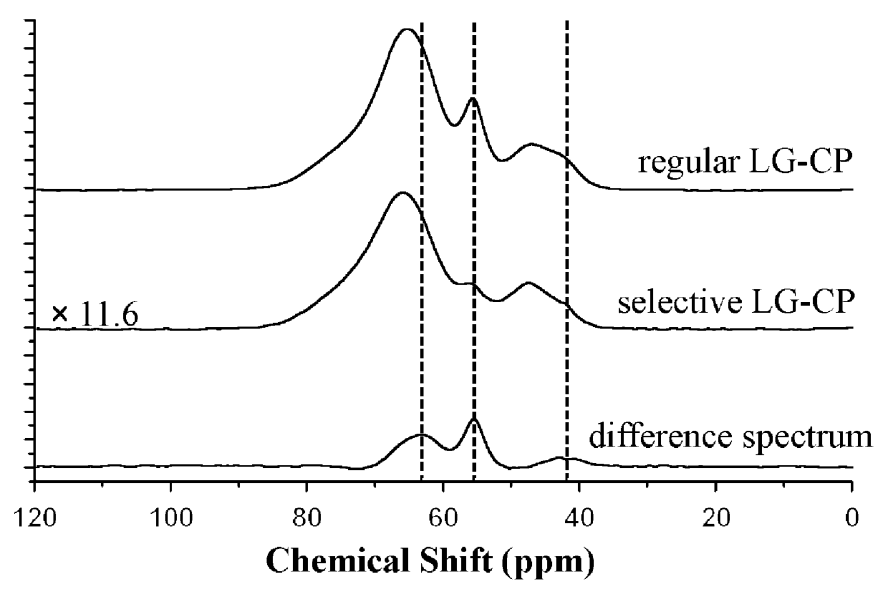

Fig. $6 .{ }^{31} \mathrm{P}\left\{{ }^{1} \mathrm{H}\right\}$ LG-CP spectra with and without selective excitation of the bridging hydroxyl group.

the difference spectrum. Signal V is the most deshielded signal in our ${ }^{31} \mathrm{P}$ spectrum, indicating that the relevant TMPO is interacting with the strongest acid site. Therefore, presumably the signal $\mathrm{V}$ is solely due to the Brønsted acid site and is hence taken as a reference signal in the difference spectrum. As a result, any signals originate from Lewis acid sites, silanol sites or Brønsted acid site with different polarization transfer dynamics will appear in the difference spectrum. Accordingly, there are two major peaks at 63 and $55 \mathrm{ppm}$ as well as a minor peak at $41 \mathrm{ppm}$ in the difference spectrum. The minor peak at $41 \mathrm{ppm}$ is readily assigned to TMPO interacting with silanol groups [17]. The peak at $63 \mathrm{ppm}$ shows that a minor portion of the signal IV in the ${ }^{31} \mathrm{P}$ MAS spectrum is due to the Lewis acid sites. It is somewhat surprising that signal II $(45 \mathrm{ppm})$ does not appear in the difference spectrum, inferring that it is due to the Brønsted acid site with weaker acidity. Because both 
Table 1

Spectral assignment of the ${ }^{31} \mathrm{P}$ MAS spectrum of TMPO-loaded HY sample

\begin{tabular}{lrll}
\hline Peak & $\%$ & Chemical shift $(\mathrm{ppm})$ & Assignment \\
\hline I & 3.5 & 41 & Physisorption/silanol site \\
II & 16.3 & 45 & Brønsted acid site \\
III & 14.8 & 55 & $\begin{array}{l}\text { Brønsted acid site (minor) } \\
\text { Lewis acid site (major) }\end{array}$ \\
IV & 55.6 & 65 & Brønsted acid site (major) \\
& & & Lewis acid site (minor) \\
V & 9.8 & 75 & Brønsted acid site \\
\hline
\end{tabular}

the signals II and $\mathrm{V}$ are not found in the $\mathrm{CP}$ difference spectrum, the signal at $55 \mathrm{ppm}$ in the difference spectrum cannot be assigned to Brønsted acid site with different polarization transfer dynamics but Lewis acid sites. Provided that the ${ }^{31} \mathrm{P}\left\{{ }^{1} \mathrm{H}\right\} \mathrm{CP}$ dynamics do not vary significantly among the acid sites, it is not unreasonable to correlate the scaled intensities of the $\mathrm{CP}$ difference spectrum with the amounts of the Brønsted and Lewis acid sites in a qualitative manner. The preliminary spectral assignment of our ${ }^{31} \mathrm{P}$ spectrum is summarized in Table 1 . However, a more quantitative analysis of the signal intensities should be avoided because of the partial selectivity of the ${ }^{1} \mathrm{H}$ soft pulse and because of the complicated $\mathrm{CP}$ spin dynamics.

In a recent communication, an LG-CP HETCOR spectrum has been measured for TMPO-loaded silica-alumina [6], where a very weak correlation peak between the bridging silanol group and the TMPO signal is observed after reintroducing ${ }^{1} \mathrm{H}$ spin diffusion before the LG-CP mixing time. In comparison, our approach has the advantage that the intense but less informative spectral component due to the methyl protons is suppressed in the spectrum.

\subsection{Effectiveness of ${ }^{1} \mathrm{H}$ selective excitation}

In this section we attempt to employ numerical simulations to study the effectiveness of ${ }^{1} \mathrm{H}$ selective excitation by a simple Gaussian-shaped pulse under the influences of ${ }^{1} \mathrm{H}$ CSA, the homonuclear dipolar interaction and the MAS frequency. The model spin system contains three protons as described in the Section 2. Referring to Fig. 7, the upper trace represents the regular MAS spectrum at $10 \mathrm{kHz}$. The middle trace shows the selective excitation of the more deshielded proton signal by a $4 \mathrm{~ms}$ Gaussian-shaped pulse under the same MAS condition. The selectively excited signal has the intensity very similar to that in the upper trace, demonstrating the satisfying selectivity. The lower trace is similarly calculated under the MAS condition of $4 \mathrm{kHz}$. A comparison of the middle and the lower traces immediately reveals the effect of MAS frequency on the selectivity of a Gaussian-shaped pulse. Additional simula-
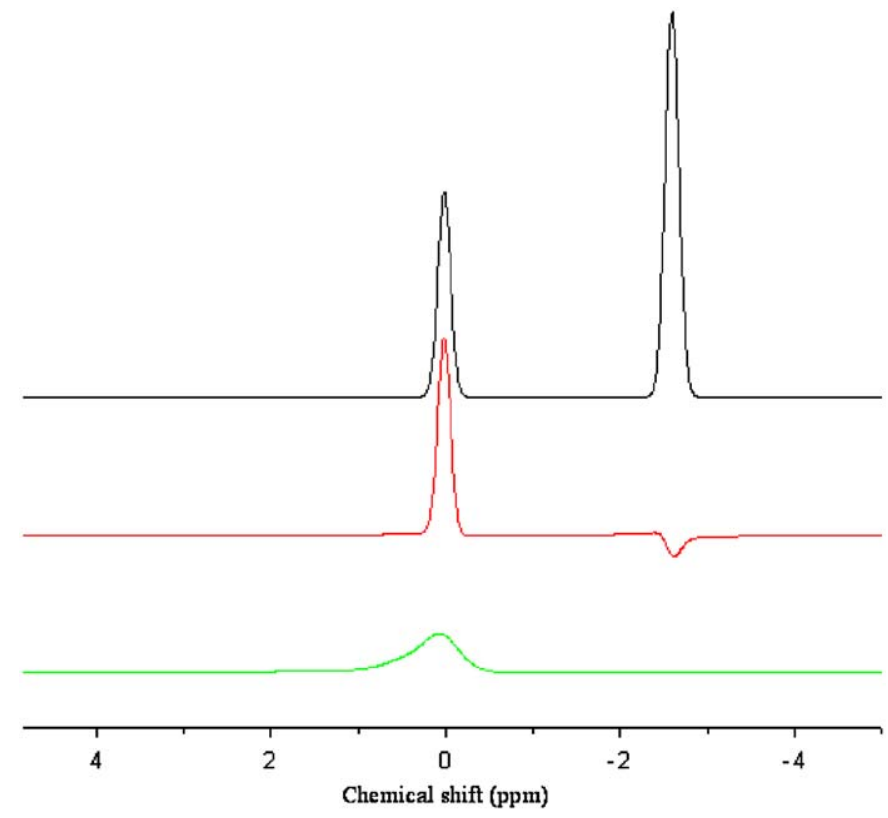

Fig. 7. Simulation spectra of a three-spin system. The upper trace is the regular MAS spectrum. The middle and the lower traces correspond to selective excitation of the deshielded proton signal under the MAS condition of 10 and $4 \mathrm{kHz}$, respectively.

tions show that selective excitation is also possible for larger dipolar interactions provided that the MAS frequency is sufficiently fast. Although the spin dynamics of the proton spin cluster in monetite is far more complicated than our three-spin model system, our results show that the MAS frequency appears to be an important experimental parameter to realize ${ }^{1} \mathrm{H}$ selective excitation.

\section{Conclusion}

We have shown that ${ }^{1} \mathrm{H}$ selective excitation can be realized by a simple Gaussian-shaped pulse for the selected samples in this study. Using selective excitation we can suppress the intense but unwanted signals in a HETCOR spectrum, or we can combine a selective pulse with LG-CP to study systems with very poor NMR sensitivity. The ${ }^{1} \mathrm{H}$ systems chosen in this study are multiple-spin systems with relatively weak ${ }^{1} \mathrm{H}-{ }^{1} \mathrm{H}$ dipolar interaction and the spectral resolution in the ${ }^{1} \mathrm{H}$ dimension is a favorable one. In other words, the ${ }^{1} \mathrm{H}$ spectra of our samples are inhomogeneously broadened when spinning at $10 \mathrm{kHz}$. We do not expect a general applicability of ${ }^{1} \mathrm{H}$ selective excitation in typical organic solids unless an adequate ${ }^{1} \mathrm{H}$ spectral resolution could be obtained at very high field and ultra-fast MAS condition $(\geqslant 45 \mathrm{kHz})$.

\section{Acknowledgments}

This work was supported by grants from the National Science Council and the Ministry of Education. We thank the detailed comments of the reviewers. 


\section{References}

[1] J. Schaefer, E.O. Stejskal, J. Am. Chem. Soc. 98 (1976) 1031-1032.

[2] A. Pines, M.G. Gibby, J.S. Waugh, J. Chem. Phys. 59 (1973) 569-590.

[3] R.A. Santos, R.A. Wind, C.E. Bronnimann, J. Magn. Reson. Ser. B 105 (1994) 183-187.

[4] B.J. van Rossum, C.P. de Groot, V. Ladizhansky, S. Vega, H.J.M. de Groot, J. Am. Chem. Soc. 122 (2000) 3465-3472.

[5] V. Ladizhansky, S. Vega, J. Chem. Phys. 112 (2000) 7158-7168.

[6] B. Alonso, I. Klur, D. Massiot, Chem. Commun. (2002) 804-805.

[7] K.S.K. Lin, Y.H. Tseng, Y. Mou, Y.C. Hsu, C.M. Yang, J.C.C. Chan, Chem. Mater. 17 (2005) 4493-4501.

[8] S.J. Huang, Q. Zhao, W.H. Chen, X.W. Han, X.H. Bao, P.S. Lo, H.K. Lee, S.B. Liu, Catal. Today 97 (2004) 25-34.
[9] Q. Zhao, W.H. Chen, S.J. Huang, Y.C. Wu, H.K. Lee, S.B. Liu, J. Phys. Chem. B 106 (2002) 4462-4469.

[10] R. Freeman, Spin Choreography: Basic Steps in High Resolution NMR, Oxford University Press, New York, 1998.

[11] M. Bak, J.T. Rasmussen, N.C. Nielsen, J. Magn. Reson. 147 (2000) 296-330.

[12] M. Bak, N.C. Nielsen, J. Magn. Reson. 125 (1997) 132-139.

[13] M. Catti, G. Ferraris, A. Filhol, Acta Cryst. B 33 (1977) 1223-1229.

[14] J.P. Yesinowski, H. Eckert, J. Am. Chem. Soc. 109 (1987) 6274-6282.

[15] C.P. Jaroniec, C. Filip, R.G. Griffin, J. Am. Chem. Soc. 124 (2002) 10728-10742.

[16] M.M. Maricq, J.S. Waugh, J. Chem. Phys. 70 (1979) 3300-3316.

[17] M.D. Karra, K.J. Stutovich, K.T. Mueller, J. Am. Chem. Soc. 124 (2002) 902-903. 